\title{
The Plastic Scintillator Detector of the HERD space mission
}

D. Kyratzis,,$^{a, b, *}$ F. Alemanno, ${ }^{a, b}$ C. Altomare, ${ }^{c, d}$ F.C.T. Barbato, ${ }^{a, b}$ P. Bernardini, ${ }^{e, f}$ P.W. Cattaneo, ${ }^{g}$ I. De Mitri, ${ }^{a, b}$ F. de Palma, ${ }^{e, f}$ L. Di Venere, ${ }^{c, d}$ M. Di Santo, ${ }^{a, b}$ P. Fusco, ${ }^{c, d}$ F. Gargano, ${ }^{d}$ F. Loparco, ${ }^{c, d}$ S. Loporchio, ${ }^{d}$ G. Marsella, ${ }^{h}$ M.N. Mazziotta, ${ }^{d}$ F.R. Pantaleo, ${ }^{c, d}$ A. Parenti, ${ }^{a, b}$ R. Pillera, ${ }^{c, d}$ A. Rappoldi, ${ }^{g}$ G. Raselli, ${ }^{g}$ M. Rossella, ${ }^{g}$ D. Serini, ${ }^{d}$ L. Silveri, ${ }^{a, b}$ A. Surdo, ${ }^{f}$ L. Wu, ${ }^{a, b}$ for the HERD collaboration

${ }^{a}$ Gran Sasso Science Institute (GSSI), Via Iacobucci 2, 67100, L'Aquila, Italy

${ }^{b}$ INFN, Laboratori Nazionali del Gran Sasso (LNGS), 67100 Assergi, L'Aquila, Italy

${ }^{c}$ Dipartimento di Fisica "M.Merlin" dell'Università e del Politecnico di Bari, 70126, Bari, Italy

${ }^{d}$ INFN, Sezione di Bari, 70126, Bari, Italy

e Dipartimento di Matematica e Fisica "E. De Giorgi", Università del Salento, 73100, Lecce, Italy

${ }^{f}$ INFN, Sezione di Lecce, 73100, Lecce, Italy

${ }^{g}$ INFN, Sezione di Pavia, 27100, Pavia, Italy

${ }^{h}$ Dipartimento di Fisica e Chimica "E. Segrè", Università degli Studi di Palermo, 90133, Palermo, Italy

E-mail: dimitrios.kyratzis@gssi.it

The High Energy cosmic-Radiation (HERD) detector is one of the prominent space-borne instruments to be installed on-board the Chinese Space Station (CSS), around 2027. Primary scientific goals regarding this initiative include: precise measurements of cosmic ray (CR) energy spectra and mass composition, at energies up to the $\mathrm{PeV}$ range; contributions to high energy gamma-ray astronomy and transient studies; as well as indirect searches for Dark Matter (DM) particles via their possible annihilation/decay to detectable products. HERD is configured to accept incident particles from both its top and four lateral sides. Owing to its pioneering design, an order of magnitude increase in acceptance is foreseen, with respect to previous and ongoing experiments. The Plastic Scintillator Detector (PSD) constitutes an important sub-detector of HERD, particularly aimed towards anti-coincidence (discriminating incident photons from charged particles), while providing precise charge measurement of incoming cosmic-ray nuclei in a range of $\mathrm{Z}=1-26$. Main requirements concerning its design, include: high detection efficiency, broad dynamic range and good energy resolution. In order to select the optimal layout, two geometries are currently under investigation: one based on long scintillator bars and the other on square tiles, with both layouts being readout by Silicon Photomultipliers (SiPMs). Ongoing activities and future plans regarding the HERD PSD will be presented in this work.

$37^{\text {th }}$ International Cosmic Ray Conference (ICRC 2021)

July 12 th - 23rd, 2021

Online - Berlin, Germany

${ }^{*}$ Presenter 


\section{Introduction}

Cosmic Rays (CRs), from their initial discovery up to the current era, constitute a significant part of scientific research concerning fundamental particle physics. A substantial amount of insight derived from the investigation of CRs, leading to an advanced understanding of the microscopic world and its intrinsic interactions.

Nowadays, CR detection remains in the forefront of intense research and is represented by a multitude of sophisticated experiments aiming towards the clarification of their origin, acceleration and propagation mechanisms in the Universe [1]. Although crucial information has been wellreceived from indirect (ground-based) experiments over the years, there is an imminent need to explore highly energetic CR particles and gamma-rays by means of direct observations, carried out by space-borne detectors. In that regard, distinct features can be revealed in the energy spectra along with a consistent picture concerning the evolution of mass composition with energy.

Results considering the interpretation of recent CR data, lead to a picture of Galactic CR nuclei being accelerated at supernova remnant (SNR) shocks by first-order Fermi mechanism and subsequently injected into the Interstellar Medium (ISM), before leaving the Galaxy [1]. That picture constitutes one of the most favorable scenarios, although conclusive results have yet to be derived. Additionally, a major unresolved issue regarding hadronic CRs is the so-called "knee", a prominent feature that marks a spectral steepening of $\mathrm{E}^{-3}$ at particle energies of few PeV. Traditionally, highly-energetic particle regions were "accessible" only by indirect CR experiments, although with an inherent difficulty in making composition studies with small systematic errors. On the contrary, direct CR experiments are capable of measuring both energy and charge of an incident particle, although due to CR fluxes' rapidly decreasing with energy (and in conjunction with limited exposure of space-borne instruments), limited statistically-meaningful results could be inferred above few tens of $\mathrm{TeV}$ with current experiments.

Consequently, forthcoming space-based experiments should incorporate requirements related to: increased geometric factor $\left(>\mathrm{m}^{2} \mathrm{sr}\right)$, extended mission duration $(\sim 5-10 \mathrm{yr})$, as well as high discrimination power in separating different cosmic radiation components. Combining the above-stated properties will result in exposure of about $15-20 \mathrm{~m}^{2} \mathrm{sr} \mathrm{yr}$, hence allowing for a deeper understanding of the intricate features that constitute distinctive structures in CR spectra.

Therefore, the High Energy cosmic-Radiation Detector (HERD) was proposed to address the aforementioned requirements, as one of the primary instruments to be installed on-board China's Space Station (CSS, as illustrated in Fig. 1a), with a planned duration of 5 to 10 years. HERD will be capable of studying spectral features of various CR nuclei with optimal precision, up to the highest-achievable energies (considering space-borne instruments), while also providing insights on various topics concerning: gamma ray astronomy, throughout a broad energy range up to several $\mathrm{TeV}$; the all-electron component and its fine structure up to $100 \mathrm{TeV}$, while distinguishing particles originating from astrophysical sources over possible dark matter annihilation products (due to inherent differences in both spectra), owing to its wide field-of-view (FoV).

An international collaboration is established around the HERD initiative, involving researchers, institutes \& universities from China, Italy, Switzerland, Spain and more countries that are willing to contribute in this endeavor. 


\section{The HERD detector}

In its baseline design [2, 3], HERD is conceived around a 3-D cubic imaging calorimeter (CALO), comprising approximately 7500 LYSO crystals that form an octagonal prism. This design ensures detection of incident particles from both its top and 4 lateral sides, as well as satisfactory energy measurements and e/p separation. Through continuous development and optimization efforts, the baseline design is upgraded with mats of Fiber Trackers (FiTs), situated on all active sides surrounding the calorimeter, in order to accurately determine tracks of impinging particles. A Plastic Scintillator Detector (PSD) is consequently covering the calorimeter and tracker, aiming to provide gamma-ray and charged particle triggers, together with an additional level of charge measurement. A further addition to the baseline design would be a Silicon Charge Detector (SCD), that envelops the above-stated sub-detectors, ensuring an additional charge determination. Finally, a Transition Radiation Detector (TRD) is placed on one of the lateral faces in order to provide energy calibration of nuclei in the $\mathrm{TeV}$ region. Consequently, an increase of more that an order of magnitude in acceptance is obtained by utilizing optimized detector techniques, in a novel design fulfilling all physics requirements, while maintaining a manageable payload for a space mission.

HERD (as illustrated in Fig. 1b) will be installed on-board the CSS (to be completed in 2022) around 2027, with a lifetime of 5-10 years. The instrument will follow a Low-Earth Orbit (LEO) at an altitude of approximately $340-450 \mathrm{~km}$, with an inclination angle of $42^{\circ}$. Meticulous placement of the instrument on the CSS, will ensure a FOV of $\pm 70^{\circ}$, while its payload will not exceed 4 tons in weight. Detailed specifications and primary scientific objectives of HERD can be found in the dedicated talk of the current ICRC edition.

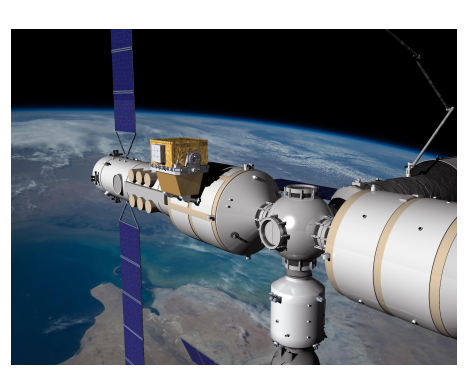

(a)

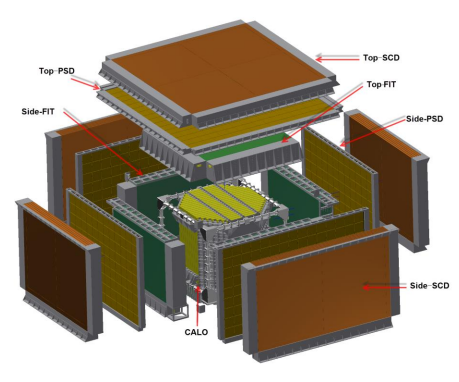

(b)

Figure 1: Graphical representation of HERD, (a) installed on the Chinese Space Station (CSS) along with (b) an exploded detector view illustrating the various sub-detectors in its most advanced design.

\section{The Plastic Scintillator Detector}

The PSD of HERD will be utilized as an anti-coincidence detector (discriminating incident photons from charged particles), while providing charge measurement of incoming cosmic-ray nuclei in a range of $Z=1-26$. Practically, thin and light materials (i.e., organic scintillators) can be an optimal choice regarding this task. Scintillator bars are readout by silicon photomultipliers (SiPMs) instead of conventional PMTs, due to recent technological developments that demonstrate: 
fast light signal detection; good sensitivity to low light yields; lower power consumption and robustness. These features delineate the practicality and versatility of SiPMs in space applications, whereas PMTs require well-increased operation voltages $(\sim \mathrm{kV})$ and lack robustness, therefore not optimized for satellite experiments. Moreover, scintillators featuring low density and good radiation hardness, while being affordable and available in mass production, seem as an intriguing choice regarding the PSD realization. Main requirements concerning its design, include: high detection efficiency, broad dynamic range and good energy resolution. Two design layouts are currently investigated, one based on long scintillator bars while the other on square (or rectangular) tiles. Both configurations present advantages and disadvantages, mainly related to the optimal number of readout channels versus back-splash (or back-scattering) effects ${ }^{1}$. Ongoing tests and optimization efforts are carried out for both configurations, aiming to define the best scintillator type and size, SiPM model and quantity to be instrumented, along with an overall verification of the PSD configuration, structural robustness and space readiness tests.

\subsection{The PSD bar option}

In its baseline design, the bar layout is composed of $170 \times 12 \times 1 \mathrm{~cm}^{3}$ scintillators regarding the top surface, while shorter bars $\left(120 \times 12 \times 1 \mathrm{~cm}^{3}\right)$ are instrumented on the lateral sides. Finally, alternating scintillator bars along the $\mathrm{X}$ and $\mathrm{Y}$ axes will be interleaved, in order to assist in track identification and charge measurement. Ongoing efforts, concern the instrumentation, calibration and performance tests of various scintillator types and SiPM models (as seen in Fig. 2). Particularly, configurations of bars with variable sizes (from $50-150 \mathrm{~cm}$ in length, $3-6 \mathrm{~cm}$ in width, 0.5 $1 \mathrm{~cm}$ in thickness) and shapes (rectangular and trapezoidal) readout by SiPMs (active area: $3 \times 3$ $\mathrm{mm}^{2}$ ) of versatile characteristics. In the following, recent measurements involving CR muons (Fig. 3a) and electrons from a radioactive ${ }^{90} \mathrm{Sr}$ source (Fig. 4) are presented, with BC-404 \& EJ-200 scintillator bars coupled with AdvanSiD or Hamamatsu SiPMs. Each contribution was fitted with a Landau-convoluted-with-Gaussian function. These tests are performed in order to validate the sensitivity of each configuration to the corresponding particle type as well as verifying inherent detector aspects concerning both scintillator (i.e., attenuation length measurement, as in Fig. 3b) $\&$ SiPM performances.

\subsection{The PSD tile option}

In the initial tile configuration [5, 6], rectangular scintillators $(10 \mathrm{~cm} / \mathrm{side} \& 0.5-1 \mathrm{~cm}$ thickness $)$ are being tested in order to cover both top and lateral faces of the instrument, adopting similar instrumentation technique to Fermi-LAT that provided satisfactory results in reducing back-splash effects. Results concerning assorted tile configurations for CR muons and electrons from a ${ }^{90} \mathrm{Sr}$ radioactive source are being shown in Fig. 5, readout by a CAEN DT5550W board coupled with CITIROC ASICs. Specifically, said configuration consists of a $10 \times 10 \times 0.5 \mathrm{~cm}^{3}$ BC-404 tile equipped with Hamamatsu SiPMs mounted on 3 PCBs. Measurements obtained from the board's Low Gain (LG) amplification channel correspond to SiPMs placed on the tile's left and top side respectively (Channels 17, 18).

\footnotetext{
${ }^{1} \mathrm{~A}$ small fraction of secondaries produced during electromagnetic showers in the calorimeter, will drift backwards and eventually reach the PSD. Electron recoils produced via Compton scattering will act as a veto for incident gammas, thus decreasing the photon detection efficiency, especially at higher energies (>10 GeV).
} 


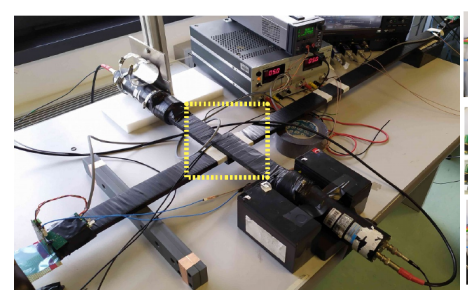

(a)

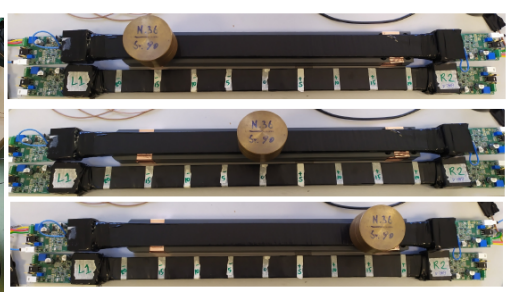

(b)

Figure 2: Geometries under investigation regarding the optimal PSD bar layout, where (a) corresponds to a BC - $404\left[150 \times 5 \times 1 \mathrm{~cm}^{3}\right]$ scintillator bar readout by 2 AdvanSiD SiPMs/side, with the addition of 2 scintillator bars readout by Hamamatsu PMTs serving as an effective trigger for CR muon measurements. For (b), an EJ - 200 [50 × $3 \times 1 \mathrm{~cm}^{3}$ ] bar instrumented with 1 Hamamatsu SiPM/side is illustrated, with a ${ }^{90} \mathrm{Sr}$ source "scanning" various positions along the tested configuration.

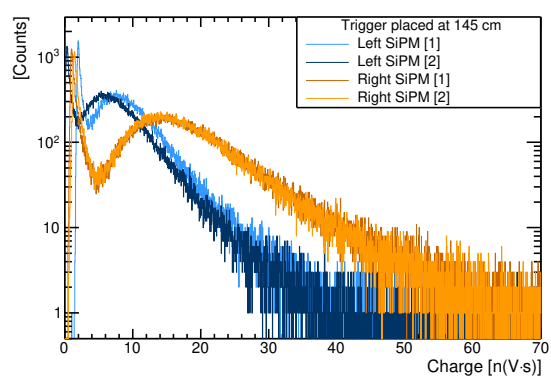

(a)

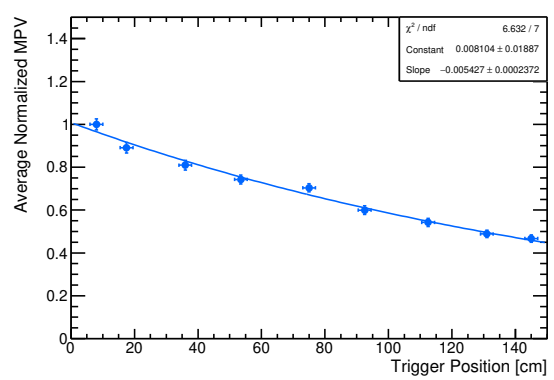

(b)

Figure 3: (a) SiPM charge distributions regarding detected CR muons in a trigger position of $145 \mathrm{~cm}$ (total bar length: $150 \mathrm{~cm}$ ) concerning a BC-404 scintillator bar, readout by 4 AdvanSiD NUV-3S SiPMs (2 SiPMs/side). (b) Calculating the average normalized Most Probable Value (MPV) from all 4 SiPMs in 9 trigger positions along the bar, the light attenuation length can be derived from the exponential fit parameters. In this specific configuration, the attenuation length corresponds to $186 \pm 13 \mathrm{~cm}$.

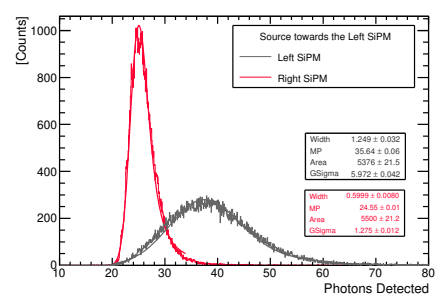

(a)

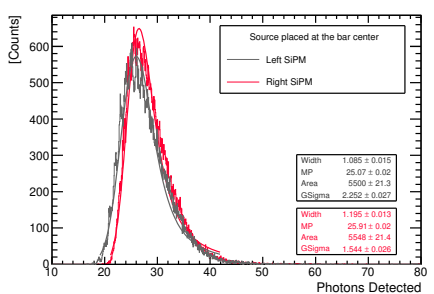

(b)

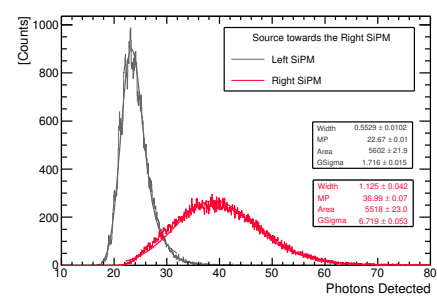

(c)

Figure 4: Spectra of photons detected regarding electrons emitted by a ${ }^{90} \mathrm{Sr}$ radioactive source in different trigger positions along a $50 \mathrm{~cm}$ EJ-200 scintillator bar, readout by 2 Hamamatsu S13360 - 3025CS SiPMs (1 $\mathrm{SiPM} / \mathrm{side}$ ). Figure (a) corresponds to a trigger position approximately $15 \mathrm{~cm}$ from the bar center, towards the left side. Additionally, (b) features charge spectra acquired with the trigger centered, while (c) reveals the symmetric trigger position $15 \mathrm{~cm}$ from the bar center, towards the right side. 
Furthermore, an additional setup was implemented with a $10 \times 10 \times 0.5 \mathrm{~cm}^{3}$ EJ-200 tile instrumented with 6 Hamamatsu S12572 SiPMs, connected in a parallel circuit and mounted on 2 PCBs instrumenting the two opposite edges of the tile. Afterwards, a series of measurements were performed by exposing the tile to proton and carbon beams at energies of $60-250 \mathrm{MeV}$ and $120-400 \mathrm{MeV} / \mathrm{u}$, respectively. Such tests were performed with a synchrotron located at the National Center for Oncological Hadrontherapy (CNAO) in Pavia (Italy), while part of the extracted measurements are illustrated in Fig. 6.

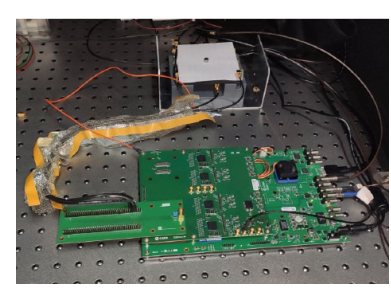

(a)

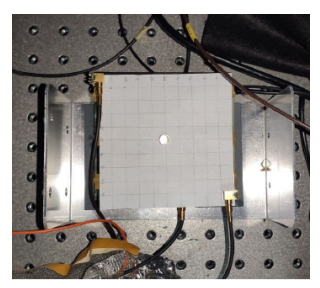

(b)

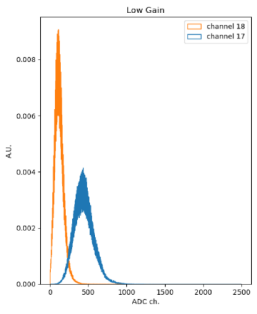

(c)

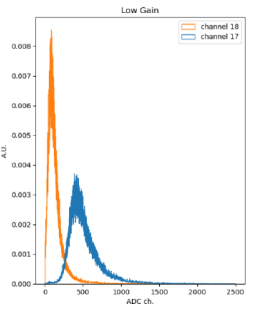

(d)

Figure 5: Tile configuration under test where (a) represents a picture of the experimental setup, (b) a closeup of the radioactive ${ }^{90} \mathrm{Sr}$ source placed on the tile, while (c) \& (d) correspond to measurements of electrons from ${ }^{90} \mathrm{Sr}$ and $\mathrm{CR}$ muons respectively, in ADC counts.

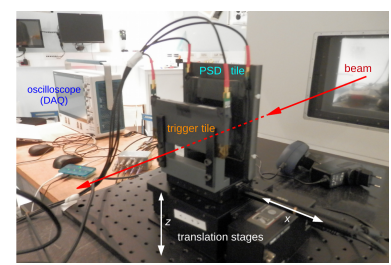

(a)

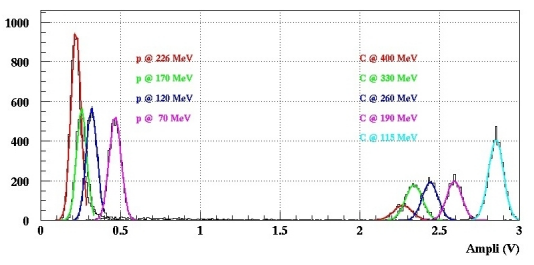

(b)

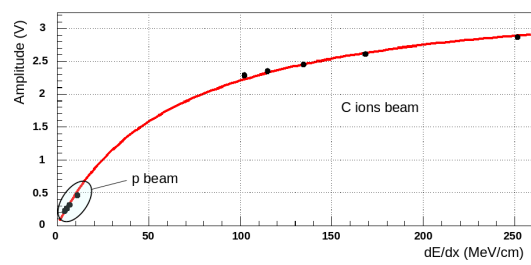

(c)

Figure 6: Tile configuration under test where (a) represents a picture of the experimental setup, (b) a signal amplitude analysis including protons and carbon nuclei in different energies, while (c) corresponds to the absolute pulse amplitude as a function of the deposited energy, extracted from proton/carbon beam data. The relation between signal amplitude and deposited energy delineates a loss of linearity that is well described by Birk's law [7] (represented by the red line).

\subsection{PSD Simulation activities}

A dedicated GEANT4-based [8] simulation software is developed with customized bar/tile layouts in order to evaluate performances and intricate detector effects of the various investigated setups along with response studies concerning a multitude of particles and nuclei. Such efforts are significant in complimenting all ongoing PSD laboratory activities and ultimately aiding in the selection between the aforementioned PSD geometries. In the following (Fig. 7) a brief example of MC simulation activities will be shown, regarding tests of tile configurations under different particle types (protons, carbon and iron nuclei) and model corrections (SiPM PDE and cross-talk) along with feasibility studies pertaining to the optimal scintillator bar shape and geometry. 


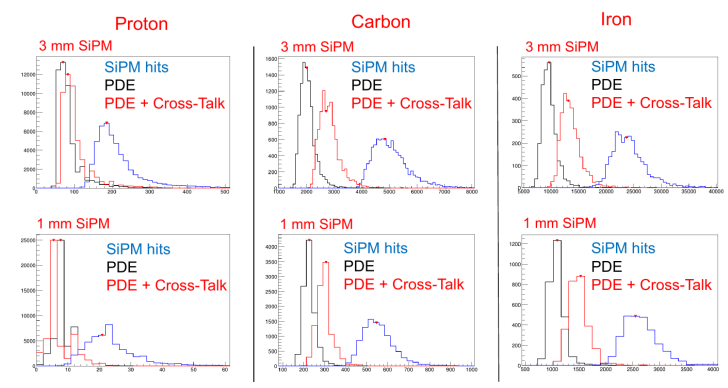

(a)

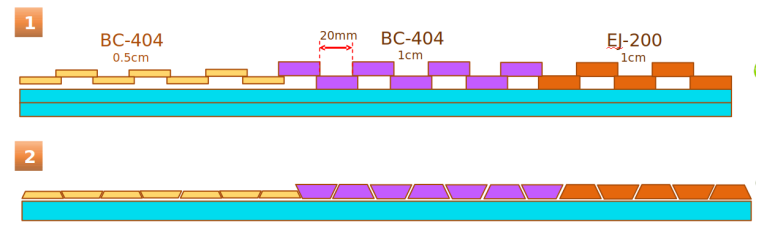

(b)

Figure 7: (a) Mean number of detected photons by 2 different SiPM sizes and 3 different particle types, applying PDE and cross-talk model corrections, for the tile configuration. (b) Hermeticity studies concerning the feasibility of novel trapezoidal bars (bottom) as opposed to default rectangular designs (top), to be verified and possibly implemented in the bar prototype of forthcoming beam test activities.

\subsection{Beam test campaign at CERN}

Of great importance in evaluating and determining the optimal PSD layout (along with all HERD sub-detectors), will be the upcoming test beam campaign, taking place at CERN SPS and scheduled for November 2021. Such an endeavor will lead to systematic performance tests of PSD bar and tile prototypes, being a collective effort of mechanical, software and hardware groups inside the collaboration. Consequently, a preliminary design corresponding to individual PSD layouts under test is illustrated below (in Fig. 8). Specifically for the tile prototype, its preliminary design includes scintillators of $10 \times 10 \times 0.5 \mathrm{~cm}^{3}$ each approximating to a sum of 25 tiles/layer and a total of 2 layers. The total prototype dimension amounts to $57.8 \times 64.5 \times 25 \mathrm{~cm}^{3}$, with a common detection area of $0.25 \mathrm{~m}^{2}$. Concerning the preliminary bar prototype, EJ-200 and BC-404 trapezoidal-shaped scintillators will be employed (in order to increase hermeticity between bars), instrumented with various Hamamatsu SiPMs (active areas: $3 \times 3 \mathrm{~mm}^{2}$ and $1 \times 1 \mathrm{~mm}^{2}$ ) covering all requested performances (high dynamic range, good linearity, high PDE, low DCR \& crosstalk). Each layer will comprise 15 bars with a total of 2 layers, while each bar will be readout by $2 \mathrm{SiPMs} / \mathrm{side}$. Total prototype dimension amounts to $61.4 \times 61.4 \times 66.8 \mathrm{~cm}^{3}$ with a common detection area of $0.25 \mathrm{~m}^{2}$.

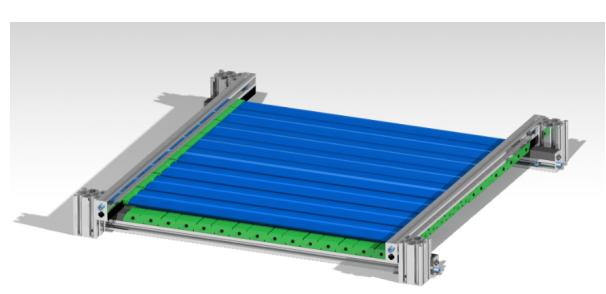

(a)

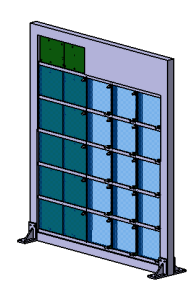

(b)

Figure 8: Preliminary designs of PSD (a) bar and (b) tile prototypes, to be validated via beam tests at CERN. 


\section{Conclusions}

The Plastic Scintillator Detector (PSD) of the HERD space mission is presented in this work, along with a brief overview of current and upcoming activities. The PSD will be employed as an anti-coincidence detector, while also providing an essential charge measurement of incoming cosmic-ray nuclei (from protons to iron nuclei). Two designs are currently under investigation: long scintillator bars and square scintillator tiles. Said options are rigorously developed from hardware and software perspectives, in order to achieve a robust understanding of the various performance aspects and inherent detector effects that arise from both designs. Main requirements of the HERD PSD include: high detection efficiency, broad dynamic range, good energy resolution as well as optimal radiation hardness and overall detector robustness, necessitated by missions in space. Numerous configurations concerning tiles \& bars have been constructed, validated and tested. In that sense, all setups were verified via a multitude of particles, ranging from: electrons, CR muons, protons and carbon nuclei in a wide energy range. Upcoming activities foresee a beam test campaign at CERN, where both prototypes will be characterized and thoroughly evaluated in order to provide insight about the optimal PSD layout.

\section{Acknowledgements}

The author would like to thank all members of the HERD collaboration for supporting this work with valuable comments and fruitful discussions.

\section{References}

[1] R. Aloisio, P. Blasi, I. De Mitri, S. Petrera, Selected Topics in Cosmic Ray Physics. In: Aloisio R., Coccia E., Vissani F. (eds) Multiple Messengers and Challenges in Astroparticle Physics. Springer, Cham. (2018)

[2] S.N. Zhang, O. Adriani, S. Albergo et al., The High Energy cosmic-Radiation Detection (HERD) facility onboard China's Space Station, Proc. SPIE, Space Telescopes and Instrumentation 2014: Ultraviolet to Gamma Ray, v. 9144, p. 91440X, (2014).

[3] D. Kyratzis, HERD: The High Energy cosmic-Radiation Detector, Nuovo Cimento C, 43, 4-5, 117, (2020).

[4] A.A. Moiseev, Observation and simulations of the backsplash effects in high-energy gammaray telescopes containing a massive calorimeter, Astroparticle Physics, v. 22(3), (2004).

[5] C. Altomare et al., Nuclear Instruments and Methods in Physics Research Section A: Accelerators, Spectrometers, Detectors and Associated Equipment, 983, 164476, (2020).

[6] P.W. Cattaneo et al, JINST, 15, C07027, (2020)

[7] J. B. Birks, Proc. Phys. Soc, A 64, 874, (1951)

[8] S. Agostinelli et al., Nuclear instruments and methods in physics research section A: Accelerators, Spectrometers, Detectors and Associated Equipment, 506, 250-303, (2003) 


\section{Full Authors List: HERD Collaboration}

O. Adriani ${ }^{26}$, F. Alemanno ${ }^{27}$, R. Aloisio ${ }^{27}$, C. Altomare ${ }^{23}$, G. Ambrosi ${ }^{34}$, Q. An ${ }^{10}$, M. Antonelli ${ }^{45}$, P. Azzarello ${ }^{37}$, L. Bai ${ }^{8}$, Y.L. Bai ${ }^{3}$, T.W. Bao ${ }^{1}$, M. Barbanera ${ }^{34}$, F.C.T. Barbato ${ }^{27}$, P. Bernardini ${ }^{30}$, E. Berti ${ }^{26}$, B. Bertucci ${ }^{35}$, X.J. Bi ${ }^{1}$, G. Bigongiari ${ }^{36}$, M. Bongi ${ }^{26}$, V. Bonvicini $^{45}$, P. Bordas ${ }^{40}$, V. Bosch-Ramon ${ }^{40}$, S. Bottai ${ }^{25}$, P. Brogi ${ }^{36}$, F. Cadoux ${ }^{37}$, D. Campana ${ }^{31}$, W.W. Cao ${ }^{3}$, Z. Cao ${ }^{1}$, J. Casaus ${ }^{39}$, E. Catanzani ${ }^{35}$, P. W. Cattaneo ${ }^{33}$, J. Chang ${ }^{9,13}$, Y.H. Chang ${ }^{21}$, G.M. Chen ${ }^{1}$, Y. Chen ${ }^{15}$, F. Cianetti ${ }^{35}$, A. Comerma ${ }^{40,41}$, D. Cortis ${ }^{28}$, X.H. Cui ${ }^{13}$, X.Z. Cui ${ }^{1}$, C. Dai ${ }^{5}$, Z.G. Dai ${ }^{15}$, R. D’Alessandro ${ }^{26}$, S. De Gaetano ${ }^{24}$, I. De Mitri ${ }^{27}$, F. de Palma ${ }^{30}$, V. Di Felice ${ }^{50}$, A. Di Giovanni $^{27}$, M. Di Santo ${ }^{27}$, L. Di Venere ${ }^{24}$, J.N. Dong ${ }^{6,7}$, Y.W. Dong ${ }^{1}$, G. Donvito ${ }^{23}$, M. Duranti ${ }^{34}$, D. D’Urso ${ }^{49}$, C. Evoli ${ }^{27}$, K. Fang ${ }^{1}$, L. Fariña ${ }^{42}$, Y. Favre ${ }^{37}$, C.Q. Feng ${ }^{10}$, H. Feng ${ }^{16}$, H.B. Feng ${ }^{5}$, Z.K. Feng ${ }^{5}$, N. Finetti ${ }^{22}$, V. Formato ${ }^{50}$, J. M. Frieden ${ }^{44}$, P. Fusco ${ }^{24}$, J.R. Gao ${ }^{3}$, F. Gargano ${ }^{23}$, D. Gascon-Fora ${ }^{40}$, D. Gasparrini ${ }^{50}$, N. Giglietto ${ }^{24}$, F. Giovacchini ${ }^{39}$, S. Gomez ${ }^{40}$, K. Gong ${ }^{1}$, Q.B. Gou ${ }^{1}$, R.

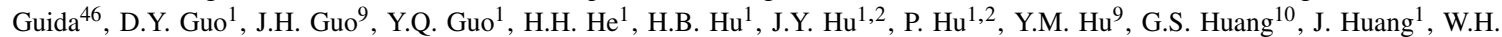

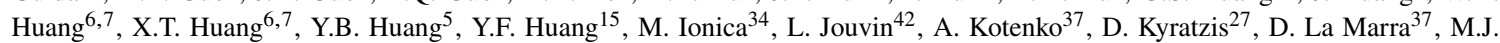

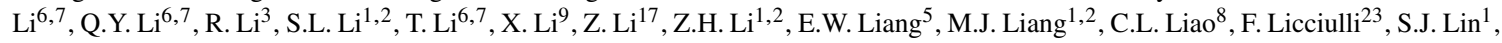

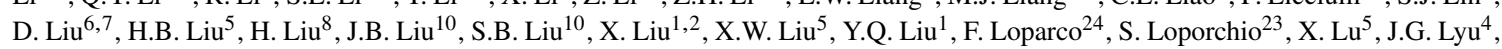
L.W. Lyu ${ }^{3}$, P. Maestro ${ }^{36}$, E. Mancini ${ }^{34}$, R. Manera ${ }^{40}$, J. Marin ${ }^{39}$, P. S. Marrocchesi ${ }^{36}$, G. Marsella ${ }^{53}$, G. Martinez ${ }^{39}$, M. Martinez ${ }^{42}$, D. Marzullo $^{47}$, J. Mauricio ${ }^{40}$, M. N. Mazziotta ${ }^{23}$, E. Mocchiutti ${ }^{45}$, G. Morettini ${ }^{35}$, N. Mori ${ }^{25}$, L. Mussolin ${ }^{35}$, A. Oliva ${ }^{51}$, D. Orlandi ${ }^{28}$, G. $^{2}$ Osteria $^{31}$, L. Pacini ${ }^{25}$, B. Panico ${ }^{31}$, F.R. Pantaleo ${ }^{24}$, S. Papa ${ }^{46}$, P. Papini ${ }^{25}$, J.M. Paredes ${ }^{40}$, A. Parenti ${ }^{27}$, M. Pauluzzi ${ }^{35}$, M. Pearce ${ }^{43}$, W.X. Peng ${ }^{1}$, F. Perfetto ${ }^{31}$, C. Perrina ${ }^{44}$, G. Perrotta ${ }^{46}$, R. Pillera ${ }^{24}$, C. Pizzolotto ${ }^{45}$, R. Qiao ${ }^{1}$, J.J. Qin ${ }^{3}$, L. Quadrani ${ }^{51,52}$, Z. Quan ${ }^{1}$, A. Rappoldi ${ }^{33}$, G. Raselli ${ }^{33}$, X.X. Ren 6 , , F. Renno ${ }^{46}$, M. Ribo ${ }^{40}$, J. Rico ${ }^{42}$, M. Rossella ${ }^{33}$, F. Ryde $^{43}$, A. Sanmukh ${ }^{40}$, V. Scotti ${ }^{32}$, D. Serini ${ }^{23}$, D.L. Shi ${ }^{3}$, Q.Q. Shi ${ }^{6,7}$, L. Silveri ${ }^{27}$, O. Starodubtsev ${ }^{25}$, D.T. Su ${ }^{12}$, M. $\mathrm{Su}^{20}$, D. Sukhonos ${ }^{37}$, A. Suma ${ }^{46}$, X.L. Sun ${ }^{1}$, Z.T. Sun $^{1,2}$, A. Surdo ${ }^{29}$, Z.C. Tang ${ }^{1}$, A. Tiberio ${ }^{26}$, A. Tykhonov ${ }^{37}$, V. Vagelli ${ }^{48}$, E. Vannuccini ${ }^{25}$, M. Velasco ${ }^{39}$, R. Walter ${ }^{38}$, A.Q. Wang ${ }^{6,7}$, B. Wang ${ }^{3}$, J.C. Wang ${ }^{14}$, J.M. Wang ${ }^{1}$, J.J. Wang ${ }^{1,2}$, L. Wang ${ }^{13}$, M. Wang ${ }^{6,7}$, R.J. Wang ${ }^{1}$, S. Wang ${ }^{9}$, X.Y. Wang ${ }^{15}$, X.L Wang ${ }^{10}$, Z.G. Wang $^{1}$, D.M. Wei ${ }^{9}$, J.J. Wei ${ }^{9}$, B.B. Wu ${ }^{1}$, J. Wu ${ }^{19}$, L.B. $\mathrm{Wu}^{27}$, X. Wu ${ }^{37}$, X.F. Wu ${ }^{9}$, Y.L. Xin ${ }^{8}$, M. Xu ${ }^{1}$, Z.Z. Xu ${ }^{10}$, H.R. Yan ${ }^{17}$, Y. Yang $^{3}$, P.F. Yin ${ }^{1}$, Y.W. Yu ${ }^{18}$, Q. Yuan ${ }^{9}$, G. Zampa ${ }^{45}$, N. Zampa ${ }^{45}$, M. Zha ${ }^{1}$, C. Zhang ${ }^{1}$, F.Z. Zhang ${ }^{1,2}$, L. Zhang ${ }^{1}$, L. Zhang ${ }^{11}$, L.F. Zhang $^{1,2}$, S.N. Zhang ${ }^{1,2}$, Y. Zhang ${ }^{9}$, Y.L. Zhang ${ }^{10}$, Z.G. Zhao ${ }^{10}$, J.K. Zheng ${ }^{3}$, Y.L. Zhou ${ }^{5}$, F.R. Zhu ${ }^{8}$, K.J. Zhu ${ }^{4}$

${ }^{1}$ Key Laboratory of Particle and Astrophysics, Chinese Academy of Sciences, Beijing, China

${ }^{2}$ University of Chinese Academy of Sciences, Beijing, China

${ }^{3} \mathrm{Xi}$ 'an Institute of Optics and Precision Mechanics of CAS, Xi' an, China

${ }^{4}$ Institute of High Energy Physics, Chinese Academy of Sciences, Beijing, China

${ }^{5}$ School of Physical Science and Technology, Guangxi University, Nanning, China

${ }^{6}$ Institute of Frontier and Interdisciplinary Science, Shandong University, Qingdao, China

${ }^{7}$ Key Laboratory of Particle Physics and Particle Irradiation, Ministry of Education, China

${ }^{8}$ School of Physical Science and Technology, Southwest Jiaotong University, Chengdu, China

${ }^{9}$ Key Laboratory of Dark Matter and Space Astronomy, Purple Mountain Observatory, Chinese Academy of Sciences, Nanjing 210023 , China

${ }^{10}$ Department of Modern Physics, University of Science and Technology of China, Hefei, China

${ }^{11}$ Department of Astronomy, Yunan University, Kunming, China

${ }^{12}$ North Night Vision Technology co. 1td., Kunming, China

${ }^{13}$ National Astronomical Observatories, Chinese Academy of Sciences, Beijing 100101, China

${ }^{14}$ Yunnan Astronomical Observatory, Chinese Academy of Sciences, Kunming, China

${ }^{15}$ School of Astronomy and Space Science, Nanjing University, Nanjing, China

${ }^{16}$ Department of Astronomy, Tsinghua University, Beijing, China

${ }^{17}$ Department of Astronomy, Peking University, Beijing, China

${ }^{18}$ Institute of Astrophysics, Central China Normal University, Wuhan, China

${ }^{19}$ Department of Physics, China University of Geosciences, Wuhan, China

${ }^{20}$ The University of Hong Kong, Hong Kong, China

${ }^{21}$ Institute of Physics, Academia Sinica, Taipei, Taiwan

${ }^{22}$ Universitá dell'Aquila and Istituto Nazionale di Fisica Nucleare, Sezione di Firenze, Firenze, Italy

${ }^{23}$ Istituto Nazionale di Fisica Nucleare, Sezione di Bari, Bari, Italy

${ }^{24}$ Dipartimento di Fisica "M.Merlin" dell'Univerisitá e del Politecnico di Bari, and Istituto Nazionale di Fisica Nucleare, Sezione di Bari, Bari, Italy

25 Istituto Nazionale di Fisica Nucleare, Sezione di Firenze, Firenze, Italy

${ }^{26}$ Universitá di Firenze and Istituto Nazionale di Fisica Nucleare, Sezione di Firenze, Firenze, Italy

27 Gran Sasso Science Institute, L’Aquila, Italy and INFN Laboratori Nazionali del Gran Sasso, Assergi, L'Aquila, Italy

28 INFN Laboratori Nazionali del Gran Sasso, L'Aquila , Italy

${ }^{29}$ Istituto Nazionale di Fisica Nucleare, Sezione di Lecce, Lecce, Italy

${ }^{30}$ Universitá del Salento and Istituto Nazionale di Fisica Nucleare, Sezione di Lecce, Lecce, Italy

31 Istituto Nazionale di Fisica Nucleare, Sezione di Napoli, Napoli, Italy

32 Universitá di Napoli "Federico II" and Istituto Nazionale di Fisica Nucleare, Sezione di Napoli, Napoli, Italy

${ }^{33}$ Istituto Nazionale di Fisica Nucleare, Sezione di Pavia, Pavia, Italy 
${ }^{34}$ Istituto Nazionale di Fisica Nucleare, Sezione di Perugia, Perugia, Italy

${ }^{35}$ Universitá degli Studi di Perugia and Istituto Nazionale di Fisica Nucleare, Sezione di Perugia, Perugia, Italy

${ }^{36}$ Universitá di Siena and Istituto Nazionale di Fisica Nucleare, Sezione di Pisa, Pisa, Italy

${ }^{37}$ Département de Physique Nucléaire et Corpusculaire (DPNC), Université de Genève, Genève, Switzerland

${ }^{38}$ Department of Astronomy, University of Geneva, Geneva, Switzerland

${ }^{39}$ Centro de Investigaciones Energéticas, Medioambientales y Tecnoló gicas (CIEMAT), E-28040 Madrid, Spain

${ }^{40}$ Dept. Física Quàntica i Astrofísica, Institut de Ciències del Cosmos (ICCUB), Universitat de Barcelona (IEEC-UB), Barcelona, Spain

${ }^{41}$ Universitat Pompeu Fabra (UPF), Barcelona, Spain

${ }^{42}$ Institut de Física d'Altes Energies (IFAE), The Barcelona Institute of Science and Technology (BIST), E-08193 Bellaterra, Barcelona, Spain

${ }^{43}$ KTH Royal Institute of Technology, Stockholm, Sweden

${ }^{44}$ Institute of Physics, Ecole Polytechnique Fédérale de Lausanne (EPFL), Lausanne, Switzerland

${ }^{45}$ Istituto Nazionale di Fisica Nucleare, Sezione di Trieste, Trieste, Italy

${ }^{46}$ Universitá degli Studi di Napoli Federico II, Napoli, Italy

${ }^{47}$ Universitá degli Studi di Trieste, Italy

${ }^{48}$ Italian Space Agency and Istituto Nazionale di Fisica Nucleare, Sezione di Perugia, Perugia, Italy

${ }^{49}$ Universitá degli Studi di Sassari and Istituto Nazionale di Fisica Nucleare, Sezione di Perugia, Perugia, Italy

${ }^{50}$ Istituto Nazionale di Fisica Nucleare, Sezione di Roma Tor Vergata, Rome, Italy

${ }^{51}$ INFN Sezione di Bologna, 40126 Bologna, Italy

52 INFN Sezione di Bologna, 40126 Bologna, Italy; Universitá di Bologna, 40126 Bologna, Italy

${ }^{53}$ Dipartimento di Fisica e Chimica "E. Segrè" Universitá degli Studi di Palermo, Palermo, Italy and INFN sez. Catania, Catania, Italy 